\title{
CONSENSO LATINOAMERICANO DE VIÑA DEL MAR
}

Entre los dias 15 y 17 de mayo fecién pasado, se celebró en la ciudad de Viña del Mar (Chile), la Reunión Extraordinaria a Nivel Mínisterial de cecta (Comisión Especial de Coordinación Latinoamericana).

El evento contó con la presencia de representantes de Argentina, Barbados, Bolivia, Brasil, Chile. Colombia, Cnsta Rica, Ecuador, E] Salvador, Guatemala, Haiti, Honduras, México, Nicaragua. Panamá, Paraguay, Perú, República Dominicana, Trinidad-Tobago, Uruguay y Venczucla. Tambien pariciparon los arganismos asesores de la Comisión: cepal., sifca y' aldalc, y observadores de Jamai Ca, BID, ILPES, ORA-CIAP Y CECIC.

Los temas dehatidus fueron los siguientes:

:. Análisis de la evolución y estado actual de la Cooperación Interamericana e Internaciona! para el desarrollo de América Latina, en función de sus planteamientos tradicionales y de los compromisos que se han generado.

11. Propuestas de América Larina.

III. Acción de América Latina para lograr sus objetivos con Estados Unidos.

En relación con ellos, el Plcnario de la Reunión aprobó por unanimidad el documento que publicamus, y que tiene su origen en los plantcamicntos elaborados por la Reunión Extraordinaria a Nivel dc Expertos, celebrada previamente a la de los Ministros de Relacinnes Extcriores.

Los paiscs miembros de la Comisión Especial de Coordinación Latinoamericana (CECLA) reunidos a Nivel Ministerial en Viña del Mar (Chile) para examinar las condiciones en que se desenvuelve la cooperación internacional y la forma como influye sobre nuestra situación externa, y proponer nuevos enfoques que respondan a la realidad del Continente, hemos acordadó la siguicnte posición común para elaborar conjuntamente con Estados Unidos de América nuevas bases para la cooperación cconómica y social interamericana.

Los Gobiernos de América Latina y el Gobierno de los Estados Unidos de América en la Declaración de los Pueblos de América, en la Carta de Punta del Este, en el Acta Económica y Social de Río de Janeiro y en la Declaración de los Presidentes de Amërica han definido obligaciones y programas de acción común que incorporan las aspiraciones de los 
ESTUDIOS INTERNACIONALES

paises latinoamericanos para alentar el desarrollo $y$ el progreso de la región. Esas obligaciones y programas no han tenido, hasta ahora, adecuado cumplimiento y atención.

Los Gobiernos de los Estados miembros de la Comisión Especial de Coordinación Latinoamericana (CECLA) reafirman la validez de los principios y propósitos contenidos en los instrumentos mencionados y la necesidad del cabal cumplimiento de los compromisos $y$ acciones especificados en los mismos.

Reiteran, además, los principios contenidos en la Carta de Alta Gracia y en la Carta del Tequendama, cuya aceptación por los Estados Unidos de América y su apoyo ante otras naciones industrializadas representarán un aporte positivo a los esfuerzos de los paises latinoamericanos destinados a lograr normas de mayor equidad en la convivencia internacional.

No obstante el hecho de que la soiución de los problemas del desarrollo ha constituido una preocupación dominante en la comunidad internacional, las decisiones, recomendaciones, principios y programas de acción hasta ahora adoptados, aun cuando constituyen un valioso acervo, no han sido suficientes. Por ello, los paises miembros de la CECLA estiman indispensable acordar formas más eficaces de cooperación interamericana einternacional.

Los conceptos que se insertan en los párrafos siguientes, que no tienen un sentido antagónico ni negativo, son la consecuencia lógica del proceso histórico de reafirmación de los valores propios de América Latina y de la toma de conciencia de sus intereses comunes.

I

\section{La .Naturaleza y el coNteximo de la cooperacióx} INTERAMERICANA E INTER.NACIONAL

1. Los paises miembros de la cECLA afirman la personalidad propia de América Latina. El proceso de desarrollo de la región y las transformacio. nes que se están operando en cada uno de sus paises, unidas a los cambios que ocurren en el mundo, imponen modificaciones importantes en las modalidades de las relaciones de América Latina con los demá: miembros de la comunidad internacional. 
Es ineludible, por tanto, que los paises latinoamericanos procuren alcanzar soluciones elaboradas con criterios propios, que reflejen su identidad nacional.

2. Decididos a superar la condición de subdesarrollo, reiteran su convicción de que el crecimiento económico y el progreso social son responsabilidad de sus pueblos y que la consecución de los objetivos nacionales y regionales depende fundamentalmente del esfuerzo de cada pais, y se sustenta también en la cada vez más estrecha cooperación, coordinación $y$ armonización de politicas $y$ actitudes de las naciones latinoamericanas, que encuentran expresión relevante en la decisión de los presidentes de los paises de América Latina de llegar a un mercado común.

3. El logro de los objetivos enunciados depende en gran medida de que la comunidad internacional $y$, en particular, los paises que hoy tienen mayor peso en las decisiones mundiales, reconozcan y asuman las responsabilidades que les corresponden.

La aceptación de esas responsabilidades y el cumplimiento de los deberes que emanan de ellas son indispensables para el más rápido y pleno aprovechamiento y movilización de los recursos internos $y$, por consiguiente, para una mayor y más completa cooperación interamer:cana e internacional que complemente el esfuerzo propio de cada país. Asimismo, contribuirán en forma importante al proceso de integración económica de América Latina.

4. Durante la última década la cooperación interamericana $y^{\prime}$ la cooperación internacional para el desarrollo de América Latina han estado lejos de satisfacer las aspiraciones de los paises de la región, tal como han sido definidas en importantes foros y documentos interamericanos $y$ mundiales. Las resoluciones, decisiones y declaraciones en la Conferencia de Bogotå de 1948; el Acta de Bogotá de 1960; la Declaración de los Pueblos de América y la Carta de Punta del Este de 196 : ; la Carta de Alta Gracia de 1964 ; el Acta Económica y Social de Rio de Janeiro de 1965; el Protocolo de Buenos Aires y la Declaración de los Presidentes de América de 1967; el Plan de Acción de Viña del Mar y la Carta del Tequendama, ambas del mismo año, y la Declaración de Santo Domingo de 1958 , inspirados en el ideal de unidad latinoamericana, han procurado impulsar de manera coherente $y$ progresiva reformas profundas de las relaciones económicas y comerciales entre la América Latina $y$ los Estados Unidos, 
E STUDIOS INTERNACIONALES

asi como entre los paises en desarrollo en general $y$ las naciones altamente industrializadas, fundadas en principios de cooperación, solidaridad, respeto a las soberanias nacionales y a la autodeterminación de ios'pucblos, y' en la necesidad de una más justa división internacional del trabajo, que lavorezca y no obstaculice, como hasta ahora, el rápido descnvolvimi ento social y económico de los países en desarrollo.

5. Al término del presente decenio, la brecha económica y científicotecnológica entre el mundo en desarrollo y las naciones desarrolladas, ha crecido y sigue creciendo y los obstáculos externos que frenan el rápido crecimiento económico de los paises latinoamericanos no sólo no han sido removidos, sino que tienden a aumentar. La persistencia de tales obstáculos se manifiesta con particular intensidad, por ejemplo, en las restricciones arancelarias $y$ no arancelarias que impiden el acceso a los grandes mercados mundiales, en condiciones equitativas o favorables, de los produclos básicos, semielaborados y manufacturados de dichos paises; en el deterioro progresivo del volumen, condiciones y modalidades de la asistencia financiera internacional, prácticamente compensada por la carga que significa cl servicio de las deudas existenies, con grave quebrantamiento de la capacidad importadora de los paises latinoamericanos; en las perturbaciones derivadas del funcionamiento del sistema monetario internacional; en las condiciones del transporte maritimo, que entraban $y$ encarecen el comercio exterior de América Latina, y en las dificultades para la transferencia de la tecnologia moderna a los países de la región, que impiden su utilización y la asimilación de ella a sus particulares necesidades, asi como la modernización de sus estructuras de producción.

6. La situación descrita exige, por una parte, el cumplimiento de los compromisos generales incluidos en la Carta de la Organización de los Estados Americanos y en el Convenio Económico de Bogotá; en el Acta de Bogotá, en la Carta de Punta del Este, y en el Acta Económica y Socia. de Rio de Janeiro, en el Protocolo de Buenos Aires y en la Declaración de los Presidentes de América; y requiere, por otra, un nuevo planteamiento de la cooperación interamericana e internacional para la realiza. ción de las aspiraciones de los paises latinoamericanos. La mayor part de estas aspiraciones ha sido identificada $y$ definida con precisión y presentada con claridad al resto del mundo. Su obiención hubiera per. mitido solucionar o evitar muchos de los problemas que han enfrenta. 
do aquellos paises, asi como sentar bases estables para la eficaz cooperación internacional.

7. Deben adoptarse medidas concretas y operativas, que se precisarán más adelante, conducentes a remover los obstáculos externos que se oponen al desarrolio acelerado de los paises de América Latina.

Tales medidas deben inspirarse en los principios ya aceptados por las comunidades interamericana e internacional $y$ que garantizan la independencia politica y económica de los paises interesados. Particularmente deben tenerse presentes los principios de igualdad juridica de los Estados; de no intervención en los asuntos internos o externos de otros Estados mediante cualquier forma que atente contra la personalidad del Estado y los elementos politicos, económicos y culturales que lo constituyen; del respeto a la validez de los tratados; del derecho soberano de cada país de disponer libremente de sus recursos naturales; de que la conperación económica no puede supeditarse a condiciones politicas o militares. Igualmente la norma de que ningún Estado podrá aplicar o estimular medidas coercitivas de carácter cconómico y político para forzar la voluntad soberana de otro Estado con el fin de obtener de éste ventajas de cualquier naturaleza $y$, por el contrario, debc hacersc todo esfuerzo a fin de evitar la adopción de políticas, acciones y medidas que pongan en peligro el desenvolvimiento económico y social de otro Estado.

8. Los principios de solidaridad que inspiran las actividades de cooperación interamericana en el campo politico y en el de la seguridad deben necesariamente aplicarse también al campo económico y social. Su no observancia en este ámbito puede perturbar la convivencia de los pueblos y poner en peligro la paz y la seguridad de las naciones.

9. La efectividad de las medidas que se han tomado y se tomen en el futuro depende en parte considerable de que los mecanismos de cooperación interamericana se adapten a los requerimientos politicos y económicos descritos y sean realmente operalivos.

Será necesario que las organizaciones y organismos de cooperación dentro de los sistemas continental y mundial agilicen su acción y la reorienten hacia los objetivos centrales del desarrollo. Asimismo, esta acción debe fundarse en el cabal conocimiento de las realidades económicas y sociales de cada país y en el respeto a las decisiones y progranas nacionales adoptados por cada Gobierno. La permanente evalua- 
ción de los programas y sus resultados es también requisito indispensable para lograr mayor eficacia en la cooperación.

10. Estos objetivos comunes deben ser complementados con la acción coordinada y eficaz de los paises latinoamericanos en los distintos foros, instituciones y organismos internacionales de cooperación de que forman parte. De esta manera la acción solidaria de América Latina tendrá mayor gravitación mundial y conducirá al logro de los objetivos perseguidos.

\section{PROPOSICIONES SOBRE MEDIDAS OPERATIVAS}

11. Basados en estos planteamientos, principios y afirmaciones, los países latinoamericanos al proponer conjuntamente un diálogo con los Estados Unidos de América deciden transmitirle sus principales aspiraciones respecto del comercio internacional, transportes, financiamiento, inversiones e invisibles, desarrollo cientifico y tecnológico, cooperación técnica y desarrollo social, con vistas a lograr, a través de las acciones y negociaciones correspondientes, un avance sólido en la cooperación interamericana. En estas áreas creen necesario:

\section{A. Comercio}

12. Insistir en el cumplimiento efectivo de los compromisos sobre statt quo, tanto en lo que se refiere a productos básicos como a manufacturas y semimanufacturas. Reiterar la nécesidad de que los mecanismos de consulta contemplados en la UNCTAD y en el GATT funcionen con ante, rioridad a la adopción de medidas que puedan significar un retrocesc en el tratamiento a la importación de los productos latinoamerica. nos. Perfeccionar tales mecanismos en el plano interamericano confor. me a la Declaración de los Presidentes de América.

13. Seguir actuando en favor de la eliminación de gravámenes aduaneros y otros obstáculos no arancelarios (tales como normas $y$-restricciones cuantitativas, de seguridad, sanitarios, etc) que afectan el acceso y comercialización de los productos básicos. Negociar con los Estados Unidos calendarios que conduzcan a la eliminación de tales restricciones en 
mercado de dicho pais para productos latinoamericanos de cspecial interës, identificando en forma conjunta la existencia de tales obstáculos. Activar la realización de una rueda de negociaciones especiales̀ en el GATT para productos básicos que no fueron debidamente tratados en lâ última rueda de negociaciones.

14. Señalar la vital importancia del cumplimiento del calendario fijado en la il uxictad sobre acuerdos de productos básicos, que incorporen disposiciones que garanticen precios equitativos $y$ remuneradores para las exportaciones latinoamericanas; el respeto de los compromisos establecidos en los acuerdos existentes; la formalización de nuevos acuerdos y ampliar, en cuanio sea necesario, su esfern de acción.

15. Revisar y' requerir la modificación y no implantación de politicas de estimulo a producciones antieconómicas de productos básicos, que perjudiquen la venta de productos latinoamericanos en los mercados mundiales y una revisión periódica de dichas politicas.

16. Desarrollar esfuerzos conjuntos para la eliminación, en un plazo percntorio, de las preferencias discriminatorias que perjudican la colocación de productos básicos latinoamericanos en ciertos mercados de paises desarrollados, sugiriendo la adopeión de medidas o acciones que faciliten e induzcan a los paises en desarrollo receptores de tales preferencias a su renuncia.

17. Demandar el efectivo funcionamiento de mecanismos de consulta en materia de colocación de excedentes y disposición de reservas, que operen respetando los principios generales ya aceptados en este campo, evitando asimismo las distorsiones en has corrientes comerciales latinoamericanas que originan los préstamos atados de la Aid $y$ la coiocación desordenada de exccdentes.

18. Revisar los sistemas bilaterales y multilaterales de asistenciat alimentaria existentes, con el propósito de ampliar sustancialmente los programas multilaterales, sobre la base de los principios aprobados en la resolución $9 / 68 \mathrm{~N}$ de la CECLA.

19. Reiterar la urgencia de que se ponga en vigor, en los plazos previstos $y^{\prime}$ respetando el calendario de reuniones programadas, el sistema de preferencias generales, no reciprocas y no discriminatorias en favor de las exportaciones de manufacturas $y$ scmimanufacturas de los paises en desarrollo. Dentro de ese esquema deberán contemplarse 
acciones que permitan a los paises de menor desarrollo económico relativo el pleno usufructo de las ventajas que resulten del mismo.

20. Eliminar, de acuerdo con un calendario fijado conjuntamente, las restricciones a la importación de productos manufacturados y semimanufacturados que interesan a América Latina, en estrecha vinculación con cl sistema de preferencias gencralcs. En esta materia, dar espccial atención al problema de la aplicación de cláusulas de escape, que requiere la adopción de critcrios y mecanismos de consulta adecuados. Evitar en este contexto la aplicación de prácticas discriminatorias de cualquicr indolc.

21. Identificar conjuntamente sectores o ramas industriales en las cuales la adopción de medidas, en plazos adecuados, por los Estados Unidos, para modificar ciertas estructuras productivas, pueda contribuir a mejorar y ampliar el comercio en cse mercado de manufacturas y scmimanufacturas de especial interés para Antérica Latina. El efecto de tales medidas scria revisádo periódicamente.

22. Reforzar, ampliar y flexibilizar, a travćs de una mayor cooperación técnica y financiera, los mecanismos nacionales y regionales de promoción de exportaciones, sistematizando la información comercial latinoamericana y buscando la colaboración de los organismos oliciales y privados de los Estados Unidos, a fin de intensificar y diversilicar las cxportaciones latinoamcricanas, facilitando asimismo los abastecimientos zonales con productos originarios del área.

23. Destacar la importancia de! apoyo activo de los Estados Unidos a los planteamientos de Latinoamérica ante otras áreas, según lo acordado en la Declaración de los Presidentes de América. El cumplimiento de sus propios compromisos por los Estados Unidos, reforzará considerablemente ei valor de dicho a poyo.

\section{B. Transporles}

24. Evitar al máximo posible que el incremento de costos operativos que se produzcan fuera del área latinoamericana, se reflejc en aumentos de los hetes maritimos que pucdan incidir en exportaciones de particular interés para los paises de América Latina.

25. Propiciar rebajas en los hetes marítimos en el comercio interameri. 
cano, cuando se produzca una reducción en los costos de operación para las cmbarcaciones en los puertos, sobre lia base del mejoramiento efectivo de cacha puerto y no de la productividad media de un corijunto de puertos.

26. Reconocer el derecho de los paises latinoamericanos de adoptar medidas de fomento en favor de sus marinas mercantes nacionales y regionales. Tales medidas de apoyo siempre que se funden en una equitaliva participación sobre las cargas que gencren las respectivas corrientes comerciales, a nivel nacional o regional según correspondá, no se considerarán discriminatorias ni porlrán dar lugar a decisiones que las anulen.

27. Ampliar la cooperación financiera y técnica interamericana, bilatcral y multilateral, para la expansión y modernización de las marinas mercantes de los paises de América Latina $y$, de acuerdo con sus propios programas, el desarrollo de su industrin naval y el mejoramiento de las instalaciones portuarias $y$ otros elementos de la infraestructura del transporte en gencral.

\section{c. Financiamiento, inversiones e invisibles}

28. La cooperación financiera interamericana, que tiene carácter complementario del esfucrzo interno, deberá regirse por los siguientes criterios básicos:

a) Constituir una transferencia real y otorgarse de acucrdo con las politicas y planes nacionales de desarrollo, ya que cllo garantizará un volumen adecuado y sostenido de recursos financieros y el derecho del pais receptor a fijar sus prioridades, mejorando la clicacia del financiamiento externo frente a situaciones que requieren un enfoque integral;

b) Los paises otorgantes $y$ las entidades financieras internacionales deben basar su coopcración en criterios económicos y socialcs, que respeten la concepción de desarrollo que tenga el pais receptor;

c) Es indispensable que la conpcración financicra externa no esté sujeta a condiciones que limiten la capacidad nacional para adoptar decisiones en el campo de las politicas cconómicas básicas del país receptor; 
E S T UDIOS INTERNACIONALES

d) Dispensar atención preferente a los paises de menor desarrollo económico relativo del área;

e) Deben suprimirse las disposiciones o criterios que ligan la utilización de préstamos a la adquisición de bienes y servicios en determinados paises o desde determinados origenes;

) Es imprescindible fortalecer una real multilateralización de la cooperación financiera externa. Los organismos financieros internacionales, en razón de su naturaleza multilateral, deben evitar que en sus decisiones influyan cventuales programas bilaterales entre paises;

g) Es necesario crear mecanismos efectivos que permitan liberalizar el crédito externo, reducir las tasas de interés y ampliar el volumen $y$ plazos de los créditos, tomando en cuenta circunstancias tales como el carácter plurianual de ciertos proyectos o programas. Proponer la creación de un Fondo de Nivelación de Intercses, cuyos recursos, asi como los que requieran otros eventuales mecanismos, deberán provenir de aportes de organismos financieros internacionales y de paises desarrollados;

h) Es conveniente una mayor participación de los organismos públicos en la canalización o utilización del financiamiento externo;

i) Se requiere adoptar providencias para que las condiciones del financiamiento externo no sean menos favorables para América Latina que para otras áreas del mundo en desarrollo.

29. Considerar indispensable la desvinculación total del financiamiento externo de elementos condicionantes, por sus múltiples efectos negativos en la economia de América Latina, tales como: la creación artificial de corrientes comerciales, inclusive las determinadas por la aplicación del criterio de la adicionalidad; el requerimiento de componente excesivo de gastos e inversiones locales, la creación de organismos superfluos, la cventual infuencia indebida en decisiones internas, el uso forzoso de determinadas lineas navicras y la realización de adquisiciones sobre la base de listas de composición inadecuadas, que representan costos elevados $y$ distorsionan el comercio de la región. Como posible solución transitoria se contenpla la utilización de fondos de créditos de la A:D u otros similares para compras en América Latina.

30. Destacar la necesidad de la reanudación del aporte financiero de los Estados Unidos a la Asociación Internacional de Fomento, y de! apoyo a América Latina para que se facilite la utilización de sus créditos por todos los paises de la región, modificando para tal fin los criterios 
Cos

de elegibilidad $y$ evitando que su otorgamiento quede vinculado a determinadas condicion es.

31. Facilitar el acceso de los paises latinoamericanos y sus organismos regionales $y$ subregionales a los mercados de capitales de Estados Unidos, mediante la disminución de sus costos y la mayor flexibilidad de los requisitos administrativos y de otra indole que lo dificultan.

32. Acrecentar las disponibilidades de fondos y mejorar la utilización de los mecanismos para el financiamiento de las exportaciones de América Latina, tomando en cuenta la necesidad de que tales créditos sean otorgados en términos y condiciones que permitan mantener y mejorar la capacidad de competencia de los productos latinoamericanos y su colocación en los mercados internacionales, incluso mediante la utilización de préstamos blandos, cuando ello dependa fundamentalmente de las condiciones financieras. En este sentido se considera importante la revisión de las condiciones de uso de los fondos del BID, de modo de ampliar los crëditos de preembarque, prever la disponibilidad de financiamiento para exportaciones de manufacturas $y$ semimanufacturas $y$ no limitarlos al comercio entre países latinoamericanos.

33. Acordar que la inversión privada extranjera no debe considerarse como asistencia ni computarse como parte de la cooperación financiera para el desarrollo. La inversión extranjera privada, sujeta a las decisiones y prioridades nacionales, debe actuar en favor de la movilización de recursos internos, generar ingresos o evitar egresos de divisas, promover el ahorro y la investigación tecnológica nacional, representar un aporte tecnológico real y participar como factor complementario de la inversión nacional, de preferencia asociada a ésta, elementos que no siempre han estado presentes. Expresar la preocupación por la magnitud global de la corriente financiera externa que ella ha originado, así como por el excesivo uso de recursos financieros locales y el efecto de ciertos acuerdos de comercialización que perturban las condiciones de competencia en los mercados internos o externos y sus posibles efectos sobre el desarrollo económico de la región.

34. Expresar su interés en el aumento de la colaboración internacional en el financiamiento de proyectos multinacionales, y extenderla al de proyectos para impulsar la integración económica, que responda a decisiones de los órganos de integración dentro de su ámbito especifico. 
ESTUDIOS INTERNACIONALE

Esta colaboración debe efectuarse de conformidad a lo expresado en la Declaración de los Presidentes de América.

35. Insistir en la necesidad de una mayor participación de América Latina en las discusiones sobre reforma del sistema monetario internacional, incluyendo aquellas que pudieran tener lugar fuera del ámbito del Fondo Monetario Internacional y particularmente dentro del liamado Grupo de los Diez. Se considera importante la pronța ratificación y activación de las disposiciones sobre Derechos Especiales de Giro y la búsqueda de mecanismos que permitan obtener en su oportunidad financiamiento adicional para el desarrollo.

36. Señalar la importancia de acrecentar el desplazamiento turistico hacia los países latinoamericanos, evitando la adopción de medidas que lo obstaculicen y apoyando con asistencia técnica y financiera el perfeccionamiento de los servicios en esta materia y' el mejoramiento de la infraestructura turistica.

37. Incluir en las revisiones anuales por paises que se efectuan en el CIAP a todos los paises que integran el sistema interamericano, a fin de examinar la ejecución de los compromisos contraidos, incluyendo aquellas politicas nacionales que pudieran incidir sobre el desarrollo económico de los paises latinoamericanos.

\section{Desarrollo social}

38. Reiterar que:

a) Su desarrollo económico debe conducir a una efectiva transformación social, cuyos objetivos básicos deben ser mejorar substanciajmente las condiciones de vida de la población, particularmente en el medio rural, y hacer participar activamente a los grupos menos favorecidos o marginados en el proceso económico y social $y$ en el pleno disfrute de sus beneficios;

b) Las inversiones para el desarrollo social son una forma de elevar los niveles de vida de la población, un factor de gran importancia para el incremento de la productividad y una mejor distribución del ingreso $y$, por tanto, merecen una atención preferente tomando en cuenta la situación particular de cada pais;

c) Los objetivos contenidos en la Declaración de los Presidentes de América sobre desarrollo social de América Latina sólo pueden tener 
completa y pronta realización si se incrementa considerablemente la coopcración internacional, técnica y linanciera para el desarrollo social, la que debe prestarse sobre la base de los programas y politicas de cada pais y contemplando sus peculiaridades nacionales.

Para esos efectos ia cooperación financiera debe otorgarse sin discriminación en condiciones especialmente flexibles, por tanto deben ser utilizados con mayor intensidad mecanismos tales como el Fondo de Operaciones Especiales del BID, cuyos recursos deben ser acrecentados oportunamentc.

\section{в. Cooperación lecnica}

39. Afirmar que los siguientes principios deben ser observados en el campo de la cooperación técnica:

a) La cooperación técnica debe ser labor conjunta de las partes interesadas. Su volumen, modalidad y forma de coordinación deben adecuarse a los objetivos nacionales de cada pais, de acuerdo con lo que cstablezcan sus planes de desarrollo económico y social;

b) La cooperación técnica debe canalizarse a través de los organismos nacionales de coordinación de cada pais y en su caso de los organismos regionales o subregionales;

c) La cooperación lécnica debc dirigirse al apoyo y' complementación de los programas nacionales de eada pais $y$ organismos encargados de su ejecución y no a la substitución de los mismos;

d) La cooperación técnica multilateral debe ser reforzada y substancialmente incrementada;

e) Emplear, en lo posible, en los programas de cooperación técnica a expertos latinoamericanos;

1) La cooperación técnica no debe disminuir a medida que los paises de América Latina alcancen etapas más avanzadis y complejas de su crecimiento, sino ser adaptada a las nuevas condiciones del proceso de desarrollo;

g) Atendiendo a las necesidades y responsabilidades que plantea el proceso de desarrollo nacional y regional, la cooperación técnica debe concederse fundamentalmente en condiciones no rcemboisables. 
40. Para el cumplimiento de sus programas de desarrollo económico $y$ social, los paises de América Latina reconocen que es necesario impulsar vigorosamente un proceso de desarrollo cientifico $y$ tecnológico, basado en el máximo esfuerzo interno y complementado con la cooperación intcrnacional. En este sentido, los paises de América Latina adoptarán una acción concertada, mediante un programa amplio de cooperación cientifica $y$ tecnológica que requiere la concurrencia de la cooperación internacional y especialmente de Estados Unidos.

41. Es. imprescindibie dar pleno cumplimiento al Programa de Acción acordado por los Presidentes de América, relativos a Ciencia y Tecnologia. Por ello y teniendo presente que el desarrollo cientifico y tecnológico exige recursos de un orden de magnitud muy superior a los que se invierten aciualmente en el plano nacional y en el regional, resulta necesario disponer de fondos especiales dedicados a tal desarrollo, los cuales debcrian otorgarse sin compromiso de retorno.

Con base en la complementación de esfuerzos que se mencionan, los Estados Unidos de América deberian:

42. Apoyar a los paises latinoamericanos en materia de ciencia y tecnologia canalizando su cooperación en función de las metas y prioridades fijadas por éstos y a través de los organismos nacionales y regionales que correspondan.

43. Adoptar métodos adecuados para mejorar la transferencia de tecnologia hacia la región. En tal sentido deberian:

a) Contribuir al mejoramiento de la información cientifica y tecnologica, mediante la capacitación de expertos y ayuda para la instalación de centros nacionales de información, los que permitirian la creación de un mecanismo regional de información científica y tècnica, incluyendo la relativa a patentes, marcas, licencias, etc.;

b) Intensificar la ayuda para mejorar la infracstructura cientificotecnológica de la región a través de, entre otras, las siguientes medidas: aumentar el intercambio de cientificos; promover programas en cooperación de investigación sobre problemas importantes para América Latina; reforzar y complementar la base fisica necesaria para las.investigaciones cientificas $y$ tecnológicas. 
44. Vejorar la transferencia de la ciencia y la tecnologia entre los paises de América Latina, para lo cual deberia:

a) Incrementar sustancialmente su apoyo financiero a los proyectos multinacionales del Programa Regional de Desarrollo Cientifico "Tecnológico;

b) Apoyar los esfuerzos de cooperación entre paises latinoamericanos en relación a enseñanza e investigación, tanto entre organizaciones estatales como privadas o universitarias.

45. Contribuir a los esfuerzos de los países de América Latina para acelerar la creación de ciencia y tecnología propias, para lo cual deberia:

a) Estimular la realización de investigaciones en los paises latinoamericanos, por las empresas estadounidenses que tienen filiales o subsidiarias en ellos, utilizando la capacidad científica y tecnológica nacional o regional;

b) Estudiar en el marco de los programas latinoamericanos, nacionales o regionales, la realización en América Latina de ciertos programas especificos de investigación científica y tecnológica de interés para la región, que actualmente realizan en Estados Unidos de América entidades estatales o paraestatales de este pais;

c) Apoyar los programas nacionales de fomento elaborados por los paises de América Latina para estimular el desarrollo cientifico y tecnológico;

d) Apoyar los esfuerzos nacionales tendientes a integrar la acción de los sectores empresarial, gubernamental, asi como universitario y de investigación tecnológica a fin de incrementar la capacidad de innovación.

46. Es necesario también acordar entre los paises de América Latina y los Estados Unidos de América una acción conjunta internacional, para favorecer el desarrollo cientifico y tecnológico de la región. En tal sentido, los Estados Unidos de América deberian:

a) Colaborar en la revisión de las convenciones internacionales vigentes sobre patentes, con el propósito de mejorar para los paises en desarrollo las condiciones de acceso a los conocimientos y procesos industriales y climinar prácticas restrictivas, permitiendo de ese modo, el empleo más eficaz de los beneficios de la ciencia y la tecnología amparados por dichos instrumentos, asi como la rápida y efectiva utiliza- 
ESTUDIOS I NTER NA G I O N A LES

ción industrial en sus territorios. La colaboración de los Estados Unidos de América en esta materia, debe comprender facilidades para un acceso más amplio, asegurando un trato equitativo y no discriminatorio a los procesos industriales sujetos a licencias $y^{\prime}$ a contratos de servicios tëcnicos. Para ello, es necesario promover en forma urgente un estudio conjunto de los problemas relativos a la transferencia $y$ absorción de tecnologia, vinculados a la institución de la patente;

b) Propugnar junto con los paises de América Latina que las instituciones financieras internacionales $y$ los organismos de créditos de los paises desarrollados, les conceden créditos en condiciones ventajosas, para la investigación cientifica y tecnológica, en el marco de las prioridades nacionales;

c) Propugnar asimismo que en los financiamientos de proyectos de desarrollo se incluyan siempre fondos para las investigaciones que ellos requieran, utilizando la capacidad cientifica $y^{\prime}$ tecnológica de los paises de la región;

d) Prestar su apoyo a la celebración (con urgencia) de una conferencia sobre la aplicación de la ciencia y la tecnologia al desarrollo latinoamericano. 